Cell Research (1999), 9 , 145--154

\title{
Comparative investigation on spindle behavior and MPF activity changes during oocyte maturation between gy- nogenetic and amphimictic crucian carp
}

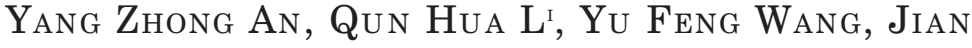 \\ FANG GU ${ }^{\text {I* }}$ \\ State Key Laboratory of Freshwater Ecology and Biotechnology, \\ Institute of Hydrobiology, Chinese Academy of Sciences, Wuhan \\ 430072, China
}

\begin{abstract}
The spindle behavior and MPF activity changes in the progression of oocyte maturation were investigated and compared with cytological observation and kinase assay between gynogenetic silver crucian carp and amphimictic colored crucian carp. MPF activity was measured by using histone $\mathrm{H} 1$ as phosphorylation substrate. There were two similar oscillatory MPF kinase activity changes during oocyte maturation in two kinds of fishes with different reproductive modes, but there existed some subtle difference between them. The subtle difference was that the first peak of MPF kinase activity was kept to a longer-lasting time in the gynogenetic silver crucian carp than in the amphimictic colored crucian carp. It was suggested that the difference may be related to the spindle behavior changes, such as tripolar spindle formation and spindle rearrangement in the gynogenetic crucian carp.
\end{abstract}

Key words: Gynogenesis, silver crucian carp, oocyte maturation, MPF kinase, tripolar spindle, meiosis.

\section{INTRODUCTION}

Silver crucian carp, Carassius auratus gibelio Bloch, is a unique triploid fish that can reproduce by a special gynogenesis[1]. In the past, cytological mechanism responsible for

\footnotetext{
* Corresponding author: e-mail:jfgui@ihb.ac.cn
} 
Spindle and MPF changes dering fish oocyte menturation

the reproductive mode had been examined in our laboratory[2]. It was found that there was no extrusion of the first polar body during oocyte maturation, which suggested that the stability of chromosomal ploidy in the gynogenetic fish might be maintained through an unusual meiosis during which there was no reduction in the genome ploidy [3].

Oocyte maturation is induced by several cell cycle mediators[4]. One of the most important factors is maturation-promoting factors (MPF) [5], a main cell cycle kinase, which is a complex of cdc2 (a catalytic subunit) and cyclin B (a regulatory subunit)[6]. MPF not only is responsible for inducing oocyte maturation but is a universal regulator of the G2/M transition in eukaryotes[4],[6]. So we assumed that the progression of oocyte maturation in the gynogenetic crucian carp might be related to the special nuclear behaviors and MPF activity changes[2],[4]. In this paper, the relationship between the spindle behavior and MPF kinase activity changes during oocyte maturation and the differences between the gynogenetic silver crucian carp and a related amphimictic subspecies colored crucian carp (Carassius auratus colored variety) were investigated and analyzed by combining cytological observation with MPF kinase assay.

\section{MATERIALS AND METHODS}

\section{Selection of brood fishes and obtainment of oocytes at different ma- turing stages}

The brood fishes of gynogenetic silver crucian carp and amphimictic colored crucian carp were selected from Guanqiao Experimental Station, Institute of Hydrobiology, Chinese Academy of Sciences. Before reproductive season, the selected brood fishes were artificially induced into maturation by two intraperitoneal injections with a mixture of acetone-dried carp pituitary, HCG and LRH-A. Acetone-dried carp pituitary was self-made. HCG and LRH-A were purchased from Shanghai Lizhu Dong Feng Biotechnology Co, LTD. The first injection was $0.6 \mathrm{mg}$ carp pituitary, $600 \mathrm{IU}$ HCG and $6 \mu \mathrm{g}$ LRH-A/kg fish weight. The second injection was $0.4 \mathrm{mg}$ carp pituitary, $200 \mathrm{IU}$ HCG and $2 \mu \mathrm{g}$ LRH-A/kg fish weight. The interval between two injections was $8 \mathrm{~h}$. The injected fishes were kept under of natural water temperature of $13-18{ }^{\circ} \mathrm{C}$. The maturing eggs at different stages were taken out with self-made fish egg taker once every $2 \mathrm{~h}$ from $0 \mathrm{~h}$ post the first injection to the moment of spawning naturally. These oocytes were washed with extrac-

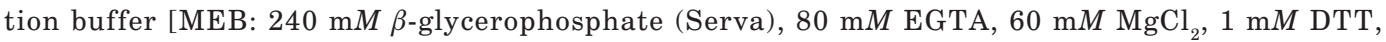
$10 \mu \mathrm{g} / \mathrm{ml}$ aprotinin (Boehringer Mannheim), $\mathrm{pH}$ 7.3]. Twenty intact oocytes were chosen to be stored at $-80{ }^{\circ} \mathrm{C}$ for following experiments, and others were fixed for cytological observations.

\section{Preparation of cell-free extracts}

The stored oocytes were crushed by homogenization at $4{ }^{\circ} \mathrm{C}$. Cold MEB was added to the oocyte homogenates, and centrifuged at $15,000 \mathrm{rpm}$ for $15 \mathrm{~min}$ at $4^{\circ} \mathrm{C}$. The supernatants were collected for further steps.

\section{Affinity chromatography of MPF kinase}

The oocyte extracts were added to $\mathrm{P} 13^{\text {suc1 }}$ sepharose beads pre-equilibrated with MEB, and vibrated for incubation of $2 \mathrm{~h}$ at $4^{\circ} \mathrm{C}$ as described previously[7]. The beads were collected by centrifugation at 15,000 rpm for 4 min and washed four times with MEB. Finally, the beads were re-suspended in MEB. The bound materials were used to analyze MPF kinase activity. 
Yang ZA et al.

\section{MPF kinase activity assay}

MPF kinase activity was assayed by using histone $\mathrm{H} 1$ as a substrate described previously[8]. Briefly, the sample of $10 \mu \mathrm{l}$ P13 beads bound with MPF kinase was incubated for 20-25 min at room temperature with $15 \mathrm{ml}$ reaction mixture [50 $\mathrm{m} M$ Tris- $\mathrm{HCl}, \mathrm{pH} 7.4,10 \mathrm{mM} \mathrm{MgCl}_{2}, 1 \mathrm{~m} M$ DTT, 2 $\mu M$ ATP, $2 \mu$ Ci $\gamma-{ }^{32} \mathrm{P}$ ATP (Furui, Peking), $2-3 \mu$ l of $2 \mathrm{mg} / \mathrm{ml}$ histone H1 (Boehringer Mannheim)]. The reaction was stopped by SDS loading buffer. The reacted samples were separated on SDSPAGE, and kinase activity was detected by autoradiography. The phosphorylated histone H1 bands were excised from the gel, and incorporated ${ }^{32} \mathrm{P}$ was quantitated by liquid scintillation count.

\section{Cytological observations}

The oocytes used for cytological observations were fixed with Bouin's solution. Serial paraffin sections were cut at 10-15 $\mu \mathrm{m}$ and stained by H.E[3].

\section{RESULTS}

\section{MPF kinase activity changes during oocyte maturation in amphimictic colored crucian carp}

In the amphimictic colored crucian carp, MPF kinase activity underwent oscillatory changes during oocyte maturation. As shown in Fig 1, the level of MPF kinase activities were very low in the oocytes taken at 0,2 and $4 \mathrm{~h}$ post-stimulation by hormones (Fig 1A). The oocytes taken at the beginning were generally at IV stage, the germinal vesicles $(\mathrm{GV})$ located in the middle. At 2 to $4 \mathrm{~h}$ of hormone injection, the germinal vesicles began to move toward the periphery (Fig 1B, 1). MPF activities were found to start to rise somewhat at $6 \mathrm{~h}$ of the stimulation (Fig $1 \mathrm{~A}$ and Fig 3). At this stage, the GV had all migrated to the animal pole (Fig 1B, 2). From 8 to 10 h, MPF activities rose quickly (Fig 1A). In the meantime, germinal vesicle breakdown (GVBD) occurred, and the maturation monaster appeared (Fig 1B, 3). The spindle formed and moved to a place near and perpendicular to the surface of the oocyte, which meant that the oocyte had entered metaphase I. While chromosomes were observed to arrange in the equatorial plate at $10 \mathrm{~h}(\mathrm{Fig} 1 \mathrm{~B}, 4)$, MPF activity reached to the first peak, and the level was about 20 times higher than that in the stage IV oocytes (Fig 1A). Thereafter, MPF activities decreased gradually, and dropped to the lowest level between two peaks at about 20 to $22 \mathrm{~h}$ of post-injection (Fig 1A). In this period, the chromosomes were first separated to two poles from metaphase equatorial plate (Fig 1B, 5). Then, the first polar body was observed to be extruded from the oocyte (Fig 1B, 6). The remained chromosomes were gradually adjusted to reform spindle (Fig 1B, 7). While the oocytes developed to metaphase II (Fig 1B, 8) at $24 \mathrm{~h}$ after the hormonal injection, the MPF activity again fast increased to the second peak. During the following $2 \mathrm{~h}$, the MPF activity level showed a little decreasing, then the eggs could be ovulated artificially. The decreased MPF activity before ovulation might be related to the disturbance caused by the continually taking out oocytes from the ovary.

We also assayed the kinase activity in the metaphase II-arrested eggs just naturally spawned from the undisturbed maternal fish, and found there existed a very high level of 
Spindle and MPF changes dering fish oocyte menturation
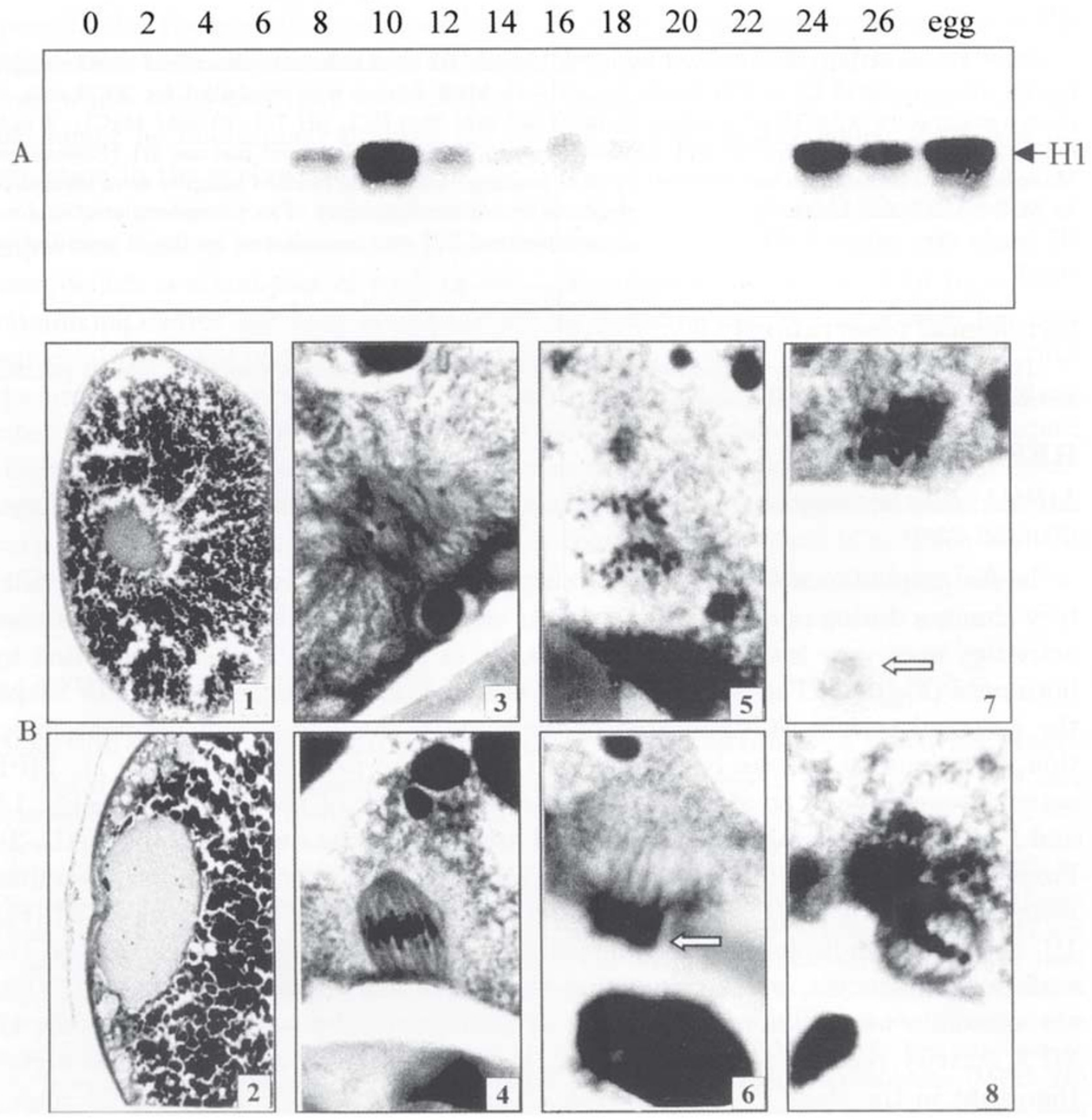

Fig 1.

MPF kinase activity and spindle behavior changes during oocyte maturation in amphimictic colored crucian carp. (A) MPF activities (histone H1 was used as a phosphorylated substrate ) in the maturing oocytes at different stages $(0,2,4,6,8,10,12,14,16,18,20,22,24,26 \mathrm{~h})$ after the hormonal injection and in the normal matured eggs (egg). (B) Cytological observation on nuclear behavior changes at different stages. (1) The GV toward the periphery in the oocyte dug out at $2 \mathrm{~h}$ after hormonal stimulation; (2) The GV just under the micropyle in the oocyte dug out at $6 \mathrm{~h}$ postinjection; (3) The maturation monaster in the oocyte dug out at $8 \mathrm{~h}$; (4) Metaphase I spindle in the oocyte dug out at $10 \mathrm{~h}$; (5) Anaphase I in the oocyte dug out at $12 \mathrm{~h}$; (6) Extruding of the first polar body (indicated by arrow) in the oocyte dug out at $16 \mathrm{~h}$; (7) Arranging chromosomes inside nucleus and the first body that had been extruded outside nucleus (indicated by arrow) in the oocyte dug out at $20 \mathrm{~h}$; (8) Metaphase II spindle in the oocyte dug out at $24 \mathrm{~h}$. 
Yang ZA et al.
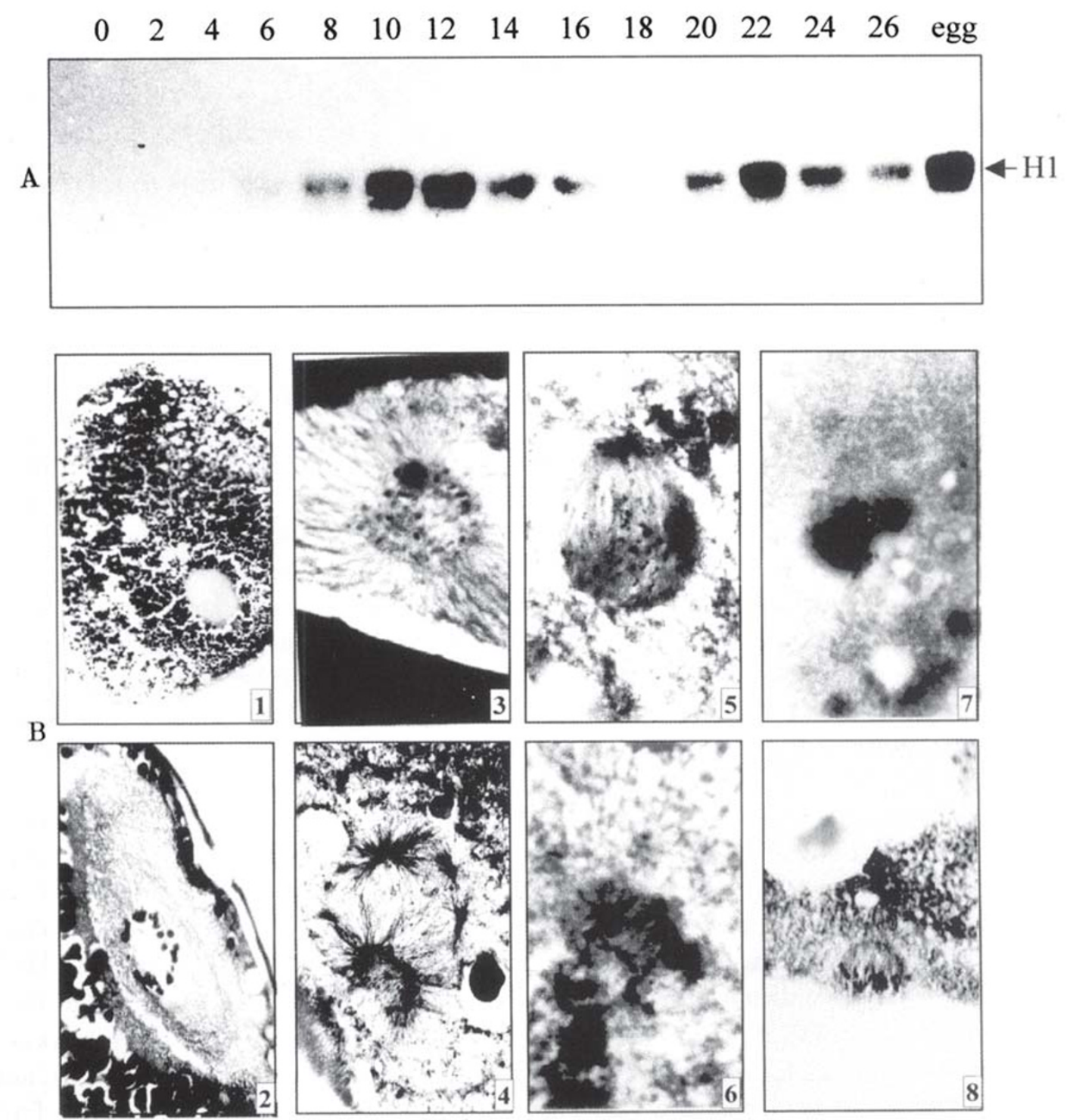

Fig 2.

MPF kinase activity and spindle behavior changes during oocyte maturation in gynogenetic silver crucian carp. (A) MPF activities (histone $\mathrm{H} 1$ was used as a phosphorylated substrate ) in the maturing oocytes at different stages $(0,2,4,6,8,10,12,14,16,18,20$, $22,24,26 \mathrm{~h}$ ) after the hormonal injection and in normal matured eggs (egg). (B) Cytological observation on spindle behavior changes at different stages. (1) GV moved outward in the oocyte dug out at $2 \mathrm{~h}$ post-injection; (2) The GV just under the micropyle in the oocyte dug out at $6 \mathrm{~h}$; (3) Maturation monaster and chromosomes in the oocyte dug out at $8 \mathrm{~h}$; (4) Maturation polyaster in the oocyte dug out at $10 \mathrm{~h}$; (5) Tripolar spindle in the oocyte dug out at $12 \mathrm{~h}$; (6) The twisting spindle in the oocyte dug out at 16 $\mathrm{h}$; (7) The bipolar-like spindle in the oocyte dug out at $20 \mathrm{~h}$; (8) Metaphase spindle in the oocyte dug out at $24 \mathrm{~h}$. 
Spindle and MPF changes dering fish oocyte menturation

MPF activity (Fig 1A).

\section{MPF kinase activity changes during oocyte maturation in gynogenetic silver crucian carp}

In gynogenetic silver crucian carp, the oscillatory changes of MPF kinase activities also occurred during oocyte maturation, but there existed subtle differences between the silver crucian carp and amphimictic colored crucian carp. As shown in Fig 2, the oocyte dug out from the un-stimulated ovary was at IV stage, and the GV was in the middle. At 2 to $4 \mathrm{~h}$ of the hormonal injection, the GV began to move outwardly (Fig $2 \mathrm{~B}, 1$ ), and migrated to the periphery at $6 \mathrm{~h}(\mathrm{Fig} 2 \mathrm{~B}, 2)$. MPF level during these stages was very low. $\mathrm{Up}$ to $6 \mathrm{~h}$ after the injection, MPF level began to rise. $2 \mathrm{~h}$ later, MPF activity increased to a higher level. At this time, GVBD began to occur in some oocytes, and the monaster could be observed (Fig 2B, 3). The first peak of MPF activity was measured in the oocytes taken out at $10 \mathrm{~h}$ following injection (Fig $2 \mathrm{~A}$ ). In the meantime, the polyaster of maturation could be observed in oocytes at this stage (Fig 2B, 4). Then, the MPF activity did not decrease so quickly like that in the amphimictic colored crucian carp, but was maintained at a longer-lasting high level in the meiosis-like metaphase I until the 12 th $\mathrm{h}$ (Fig 2A). During this period, the polyaster of maturation gradually gathered together and rearranged to form tripolar spindle (Fig 2B, 5). By 14-16 h post injection, the tripolar spindle began to twist and overlap (Fig 2B, 6), and prepared to re-unite into a bipolar-like spindle (Fig 2B, 7). During the period, the MPF activities gradually decreased, and decreased to a low level at about 18 to $20 \mathrm{~h}$. At about $22 \mathrm{~h}$ of the hormonal injection, when MPF kinase activity again fast increased to a higher level (Fig 2A), normal bipolar spindle had formed and moved to the edge of the oocyte, and the chromosomes were regularly arranged on the equatorial plate of a typical metaphase (Fig 2B, 8). Like the case as observed in the amphimictic colored crucian carp, MPF activity levels also displayed a little decrease during a few hours before ovulation.

Additionally, we also detected the kinase activity in the normal eggs spawned naturally from the female that did not artificially disturbed by digging oocytes, and found that there also existed a very high level of MPF activity (Fig 2A). Therefore, the decrease of MPF kinase activity in the oocytes at later stages was again demonstrated to be related to the artificial disturbance.

\section{Comparative analysis on MPF kinase activity changes during oocyte maturation between the gynogenetic and amphimictic crucian carp}

In order to compare the similarities and differences of MPF kinase activity changes during oocyte maturation between gynogenetic silver crucian carp and amphimictic colored crucian carp, we further analyzed the statistical data from three repeated experiments (Fig 3). As shown in Fig 3, there were two similar oscillatory changes of MPF kinase activity during oocyte maturation in two kinds of carps with different reproductive 
Yang ZA et al.

modes, but there existed a subtle difference between them which involved a longer-lasting first peak of MPF kinase activities in gynogenetic silver crucian carp than that in amphimictic colored crucian carp. It was suggested that this difference might be related to the tripolar spindle formation and its rearrangement.
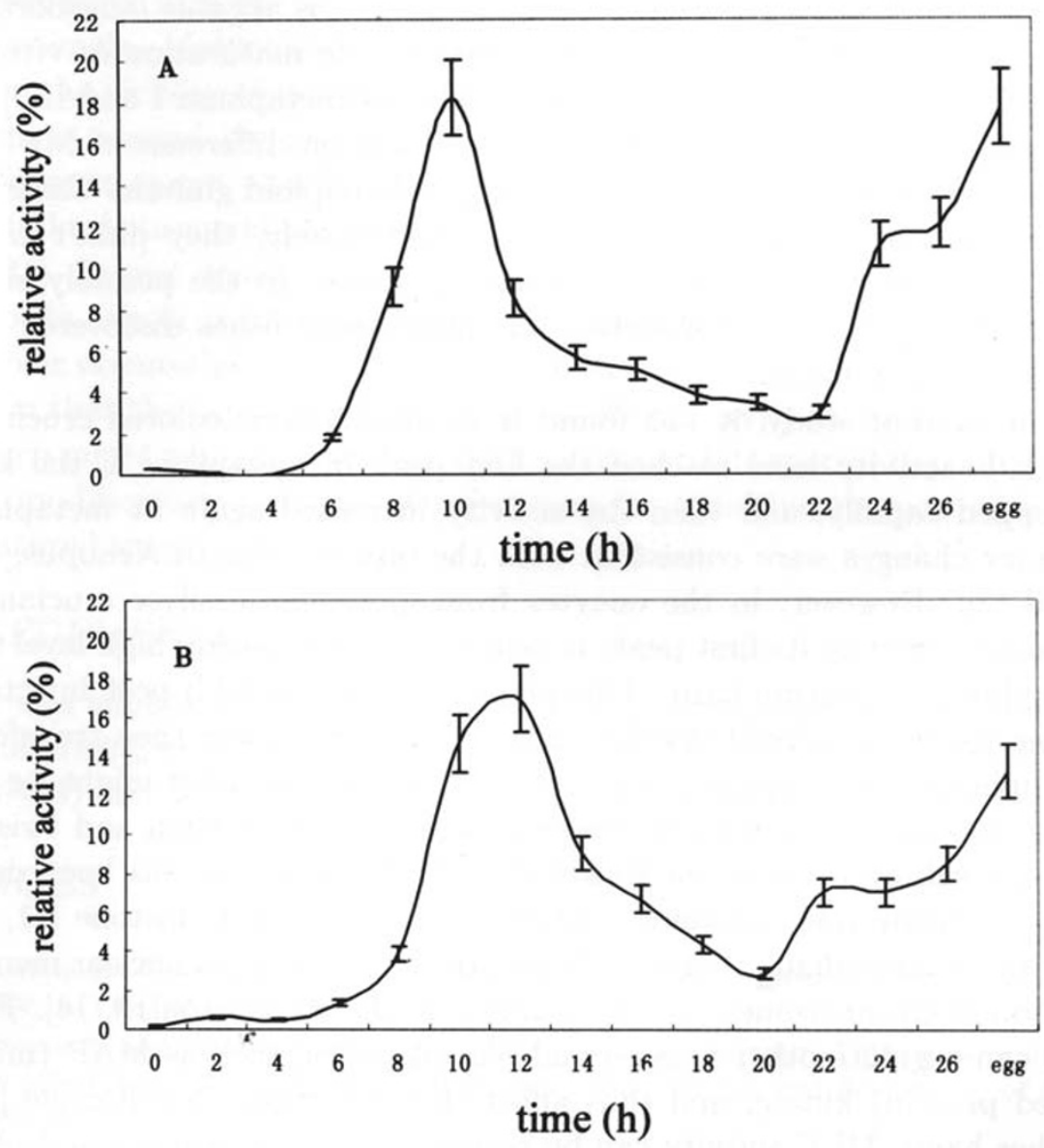

Fig 3.

Statistical comparison on relative MPF kinase activity during oocyte maturation at different stages $(0,2,4,6,8,10,12,14,16,18,20,22,24,26 \mathrm{~h})$ after hormonal injection and in normal matured eggs (egg) between amphimictic colored crucian carp (A) and gynogenetic silver crucian carp (B). The data were statistical results from three repeated experiments.

\section{DISCUSSION}

It is obvious from this study and previous report[3] that the changes of spindle behavior during oocyte maturation in the gynogenetic silver crucian carp were significantly different from that in the amphimictic colored crucian carp. The chromosomes were first ar- 
ranged to become a tripolar spindle, then the tripolar spindle was turned round and reunited mutually, and finally a normal bipolar spindle was formed. Din and Jiang once thought that some cytoplasmic factors might play an important role in the progress of tripolar spindle formation and bipolar spindle transformation[3].

In another gynogenetic triploid ginbuna Carassius auratus langsdorf, Yamashita et al. had assayed the MPF activity during oocyte maturation in vitro and found that MPF activity decreased transiently between metaphase I and II[9]. According to this observation, they thought that there was no difference of MPF activity in the meiotic progression between the gynogenetic triploid ginbuna Carassius auratus langsdorf and other bisexual animals. Unfortunately, they didn't carry out the control experiment with related amphimictic fishes, so the possibly similar subtle difference between the gynogenetic and amphimictic fishes discovered by us might not be noticed by them.

In the present study, it was found from amphimictic colored crucian carp that after MPF activity level reached the first peak in metaphase I, the kinase activity dropped rapidly, and then the activity increased again at metaphase II. The oscillatory changes were consistent with the observations in Xenopus, starfish and mice[10-12]. However, in the oocytes from gynogenetic silver crucian carp, after MPF activity got to its first peak, it kept at a longer-lasting high level than that in the amphimictic crucian carp. This period between 10-12 h post injection was just the time when the special tripolar spindle was formed and then transformed. This may implicate that the extra high of level of MPF activity might be contributed to some special events, such as the tripolar spindle formation and twisting. As an important cell cycle regulator from G2 to M, MPF kinase has been demonstrated to phosphorylate some proteins related to mitosis, such as histone $\mathrm{H} 1$, lamins, $13 \mathrm{~S}$ condensin, intermediate fiber etc, leading to the breakage of nuclear membrane, condensation of chromosomes, and rearrangment of cytoskeleton[13],[14]. Furthermore, it also can regulate other kinases and phosphatases, such as MAP (microtuble associated protein) kinase, and thus affect their functions in cell cycle [14], [15]. On the other hand, MPF activity can be regulated by other kinases and phosphatases, such as Wee1 and cdc 25[16-18]. So the level of MPF activity directly affect the progress of events occurred during cell cycle. Masui and Markert and Kishimoto et al. had reported respectively that when MPF was microinjected into G2-arrested frog or starfish oocytes, the transition from G2 to M occurred[5],[19]. While artificially degrading MPF in mouse oocytes could cause chromosomes moving toward two poles which usually occurred at postmetaphase[12]. So MPF might influence the formation and arrangement of microtubles by cooperating with other kinases and/or phosphatases, and thereby leading to the formation and twisting of tripolar spindle.

It was established that MPF kinase consists of $\mathrm{p} 34^{\mathrm{cdc} 2}$ and cyclin $\mathrm{B}[20]$. When cells entered $\mathrm{M}$ phase, $\mathrm{p} 34^{\mathrm{cdc} 2}$ dephosphorylated and combined with a newly synthesized cyclin B to resume its activity. In the meantime, the cyclin B was phosphorylated and the kinase was activated, thus the cell could come to $M$ phase. When cells left $M$ phase, the cyclins were rapidly degraded and MPF activity disappeared, which made the transition 


\section{Yaargg ZIAA et al.}

from M to G1[13]. So, we hypothesized that the first long-lasting peak of MPF activity in the gynogenetic silver crucian carp might be due to the possibility that the cyclin degradation may not the same as that in the amphimictic animals. Maldonado-Codina et al. (1992) revealed that cyclin B was seen mainly in the vicinity of microtubules throughout the mitotic cycle and it was most conspicuous around the centrosomes. Therefore, they thought that cyclin B might act in vivo to target $\mathrm{p} 34^{\mathrm{cdc} 2}$ kinase to the astral microtubles where it would play an essential role in modifying microtubule dynamics upon the formation of mitotic spindle[21]. Recent studies showed that microtubule dynamics at kinetochores, in addition to its role in moving chromosomes, might reflect the mechanisms used to ensure bipolar orientation of chromosomes[22]. These opinions further supported the assumption that the long-lasting high level of MPF activity in the oocytes of silver crucian carp might be responsible for the formation of its special tripolar spindle and the later modification of microtuble dynamics for reuniting the normal bipolar spindle at matured stage.

\section{ACKNOWLEDEMENTS}

This work was supported by the National Natural Science Foundation of China (Grants No. 39570363 and 39730290), the Chinese Academy of Sciences (Grant No. KJ951B1-607-03) and Wuhan Dawn Plan (Grant No. 975006090).

\section{REFERENCES}

[1] Gui J-F. A unique study system: gynogenetic fish Carassius auratus gibelio. Science Foundation in China, 1996; 4(1):44-6.

[2] Gui JF. Retrospects and prospects of studies on the mechanism of natural gynogenesis in silver crucian carp (Carassius auratus gibellio). Bulletin of National Natural Foundation of China, 1997; 11(1):11-6.

[3] Din J, Jiang Y-G. Comparative cytological studies on the oocyte maturation in gynogenetic crucian carp and amphimictic red carp. Acta Hydrobiologica Sinica, 1991; 15(2):97-102.

[4] Gui J-F. Roles of oocyte in studies on the cell cycle regulation. Bulletin of National Natural Foundation of China, 1996; 10(3):173-8.

[5] Masui Y, Markert CL. Cytoplasmic control of nuclear behevior during meiotic maturation of frog oocytes. J Exp Zool 1971; 177:129-45.

[6] Masui Y. A quest for cytoplasmic factors that control the cell cycle. In: Progress in Cell Cycle Research (Meijer L, Guidet S, Vogel L, eds.), Plenum Press, New York USA 1996; Vol 2, 1-13.

[7] Pfaller R, Smythe C, Newport JW. Assembly /disassembly of the nuclear envelope membrane: cell cycle-dependent binding of nuclear membrane vesicles to chromatin in vitro. Cell 1991; 65:209-17.

[8] Gui J-F, Lane WS, Fu X-D. A serine kinase regulates intracellular localization of splicing factors in the cell cycle. Nature 1994; 369:678-82.

[9] Yamashita M, Jiang $\mathrm{J}$ et al. A tripolar spindle formed at meiosis I assures the retention of the original ploidy in the gynogenetic triploid crucian carp, Giinbuna Carassius auratus langsdorfii. Dev Growth Differ 1993; 35(6):631-6.

[10] Jerhart J, Wa M, et al. Cell cycle dynamics of an M-phase-specific cytoplasmic factor in Xenopus laevis oocytes and eggs. J Cell Biol 1984; 98:1247-55. 